\title{
Sobre os autores / autoras desta edição:
}

\section{Glaucia Mayara Niedermeyer Orth}

Mestre e Doutora em Ciências Sociais Aplicadas pela UEPG. Bacharel em Psicologia pela UNICENTRO. Psicóloga da Fundação de Assistência Social de Ponta Grossa-PR. Professora no Departamento de Direito e coordenadora da pós-graduação lato sensu de ?Justiça Restaurativa e Mediação de Conflitos? na UNISECAL. Email: glauciamno88@gmail.com

\section{Jussara Ayres Bourguignon}

Mestre e Doutora em Serviço Social pela PUC-SP. Bacharel em Serviço Social pela UEPG. Professora Adjunta do Departamento de Serviço Social e do Programa de Pós-Graduação em Ciências Sociais Aplicadas da UEPG. Email: juaybo@gmail.com

\section{Dirceia Moreira}

Mestre e Doutora em Direito das Relações Sociais pela PUC-SP. Bacharel em Direito pela UEPG. Professora Adjunta do Departamento de Direito e do Programa de Pós-Graduação em Ciências Sociais Aplicadas da UEPG. Email: dirceiam@gmail.com

\section{Ana Paula Pereira Flores}

Advogada. Mestra em Serviço Social pelo Programa de Pós-Graduação em Serviço Social da Escola de Humanidades da Pontifícia Universidade Católica do Rio Grande do Sul (PUCRS). Assessora do Programa Justiça para o Século 21, do Tribunal de Justiça do Estado do Rio Grande do Sul de Abril de 2015 até Agosto de 2018.

\section{Débora Eisele Barberis}

Mestre em Direito Político e Econômico pela Universidade Presbiteriana Mackenzie com tema da Justiça Restaurativa. Advogada, Mediadora, Facilitadora e Formadora de facilitadores de Processos Circulares. Professora do Curso de Formação de Facilitadores promovido pelo TJ (SP). Facilitadora no curso de Fundamentos da Justiça Restaurativa do CDHEP.Email:deboraebarberis@hotmail.com 


\section{Fernanda Yumi Furukawa Hata}

Juíza de Direito do Tribunal de Justiça do Estado de São Paulo (TJSP). Mestre em Direito Penal pela Pontifícia Universidade Católica de São Paulo (PUCSP). Especialista em Direito Penal pela Universidade de Salamanca (ESP). Formação em Princípios, Teoria e Aplicações da Justiça Restaurativa pela Eastern Mennonite University (EMU/EUA). Facilitadora em Justiça Restaurativa pela Escola Superior da Magistratura do Rio Grande do Sul (AJURIS). Fundadora do Núcleo de Estudo e Práticas Restaurativas de Socorro (NEPRES). Facilitadora em Constelação Familiar. Discente do curso de Psicologia pela Universidade São Francisco (USF). E-mail: fernandayumi@yahoo.com.br

\section{Diego Dall’Agnol Maia}

Advogado. Mestrando em Filosofia do Direito pela Pontifícia Universidade Católica de São Paulo (PUCSP). Especialista em Direito Público com ênfase em Direito Administrativo e Direito Constitucional pela Pontifícia Universidade Católica de Campinas (PUCCAMP). Formação em Princípios, Teoria e Aplicações da Justiça Restaurativa pela Eastern Mennonite University (EMU/EUA). Facilitador em Justiça Restaurativa pela Escola Superior da Magistratura do Rio Grande do Sul (AJURIS). Membro do Fórum Europeu para Justiça Restaurativa (EFRJ). Voluntário no Núcleo de Estudo e Práticas Restaurativas de Socorro (NEPRES).E-mail: ddam.diego@gmail.com

\section{Arthemis Nuamma Nunes de Almeida}

Mestre em Psicologia pelo programa de Pós-Graduação em Psicologia da Universidade Federal do Rio Grande do Norte (UFRN). Psicóloga no Centro-dia de referência para pessoa com deficiência em Natal/RN. Membro da Comissão de Educação do Conselho Regional de Psicologia - CRP/17. E-mail: arthemisnuamma7@gmail.com.

\section{Ilana Lemos de Paiva}

Professora do Departamento de Psicologia da Universidade Federal do Rio Grande do Norte e Coordenadora do Observatório da População Infantojuvenil em Contextos de Violência (OBIJUV). E-mail: ilanapaiva@hotmail.com. 


\section{Ana Ludmila Freire Costa}

Professora adjunta do curso de Psicologia da Universidade Federal do Rio Grande do Norte (UFRN), lotada na Faculdade de Ciências da Saúde do Trairi. Possui graduação (2004), mestrado (2006), doutorado (2014) e estágio pós-doutoral (2015-2017) pela UFRN. E-mail: analudmila@gmail.com.

\section{Ana Lyvia Aragão Martins}

Licenciada em Ciências Socias pela Universidade Estadual do Ceará - UECE; pesquisadora do Laboratório de Conflitualidade e Violência - COVIO/UECE e do Laboratório de Práticas Sociais - Lapráticas/UECE. E-mail: lyvinhah@gmail.com.

\section{Maria Cristiane Lopes da Silva}

Mestranda do Programa de Pós-Graduação em Sociologia pela Universidade Estadual do Ceará - UECE; Licenciada em Filosofia - UECE e bacharel em Serviço Social - UECE; Professora da SEDUC-CE; Pesquisadora do Laboratório de Conflitualidade e Violência - COVIO/UECE e do Laboratório de Práticas Sociais - Lapráticas/UECE. E-mail: crisneto19@gmail.com.

\section{Rosemary de Oliveira Almeida}

Doutora em Sociologia pela Universidade Federal do Ceará -UFC. Pesquisadora do Laboratório de Estudos da Conflitualidade e Violência/COVIO/UECE e do Laboratório de Práticas Sociais - Lapráticas/UECE. E-mail: rosemary.almeida@uece.br.

\section{Josineide Gadelha Pamplona Medeiros}

Juíza de Direito do Tribunal de Justiça do Estado do Pará. Mestre em Direito, com ênfase em Direitos Humanos, pela Universidade Federal do Pará (UFPA). Doutoranda pelo Programa de Pós-graduação em Sociedade, Natureza e Desenvolvimento da Universidade Federal do Oeste do Pará (UFOPA). Membro do Comitê Gestor da Justiça Restaurativa do Conselho Nacional de Justiça (CNJ). 


\section{Nirson Medeiros da Silva Neto}

Professor Adjunto da UFOPA. Possui pós-doutorado pelo Departamento de Psicologia Social e do Trabalho, Instituto de Psicologia, da Universidade de São Paulo (USP), doutorado em Ciências Sociais, área de Antropologia, e mestrado em Direito, com ênfase em Direitos Humanos, pela UFPA. Atualmente, é membro do Programa de Pós-graduação em Ciências da Sociedade (PPGCS), coordenador da Clínica de Justiça Restaurativa da Amazônia (CJUÁ) e vice-diretor do Instituto de Ciências da Sociedade (ICS) da UFOPA.

\section{Maria de Jesus Pedreira Ferreira}

Licenciatura plena em Pedagogia, com Especialização em Educação e Problemas Regionais; Pedagoga no Ensino Fundamental e Médio da rede pública estadual-AP. E-mail: jesuspedreira1@hotmail.com.

\section{Girlene Bacelar Lima}

Licenciatura plena em Ciências Biológicas; Pós-graduada em Perícia Ambiental; Professora de Ciências no Ensino Fundamental da rede pública municipal e estadual-AP. E-mail: girlene-bacelar@bol.com.br.

\section{Rute Helena da Silva Gama}

Licenciatura plena em Pedagogia, com Especialização em Pedagogia Escolar; Professora no Ensino Fundamental da rede pública estadual-AP. E-mail: r-helenagama@bol,com.br.

\section{Marisa Martins Cardoso}

Acadêmica do Curso de Especialização da Universidade Federal do Pará. Professora colaboradora da Secretaria de Educação do Município de Bragança.

\section{Ana Paula Vieira e Souza}

Doutora em Educação e Professora da Universidade Federal do Pará, da Pós-Graduação em Linguagens e Saberes da Amazônia do Campus Universitário de Bragança. Membro 
do Grupo de Estudos e Pesquisa sobre Trabalho e Educação (GEPTE), coordenadora da Sessão Trabalho e Infâncias/UFPA. paulladesa@ufpa.br.

\section{Rodrigo Fraga Garvão}

Mestre em Desenvolvimento e Meio Ambiente urbano pela universidade da Amazônia (UNAMA), Professor da Universidade Federal Rural da Amazônia (UFRA), email:rodrigofragabh@gmail.com

\section{Ketno Lucas Santiago}

Acadêmico do Mestrado do Programa de Pós-Graduação em Linguagens e Saberes da Amazônia - PPLSA/ Campus Bragança (UFPA). Especialista na Docência do Ensino Superior, Historiador. Filiado a ANPH - Brasil, Membro do GELCONPE (Grupo de Estudos de Literatura Comparada do Nordeste Paraense) e do GEPTE/Infância. E-mail: ketnolucas@gmail.com.

\section{Francisco Pereira Smith Júnior}

Professor Doutor Adjunto III (UFPA). Professor do Programa de Pós-graduação em Letras, Linguagens e Saberes na Amazônia (PPLSA). Coordenador do Grupo de Estudos de Literatura Comparada do Nordeste Paraense - GELCONPE. E-mail: fsmith@ufpa.br.

\section{Ana Paula Vieira e Souza}

Professora Doutora em Educação (UFPA). Professora do Programa de Mestrado em Linguagem e Saberes da Amazônia, (PPLSA-UFPA). Coordenadora do GEPTE/Infância. Email: paulladesa@ufpa.br

\section{Givanilton de Araújo Barbosa}

Mestrando em Antropologia Social, Licenciado em Ciências Sociais e Membro do Grupo Interdisciplinar de Pesquisa em Cultura, Sociedade e Ambiente todos pela UFPB. givaniltonbarbosa10@gmail.com. 


\section{Tomás Cajueiro}

Tomás Cajueiro é Bacharel em Ciências da Comunicação pela Universidade para Estrangeiros de Perugia, Itália, e Mestre em Política Internacional pela Universidade de Manchester, Reino Unido. Conclui, em 2016, um MBA em Gestão Cultural na Fundação Getúlio Vargas, São Paulo. Atua no Brasil e no exterior como fotógrafo e produtor cultural desenvolvendo projetos de fotografia documental com forte cunho etnográfico. tomas@cajueiroproducoes.com.br

\section{Thiago Guimarães do Sacramento}

Mestrando do Programa de Pós-Graduação de Ciência da Sociedade da Universidade Federal do Oeste do Pará. 